\title{
K-CORRECTION BIASES AND THE QUASAR LUMINOSITY FUNCTION
}

\author{
A. C. BAKER and P. C. HEWETT \\ Institute of Astronomy, Madingley Road, Cambridge, CBS OHA England
}

\begin{abstract}
.
By characterising the range of quasar UV-optical spectral indices and any correlation with it e.g. luminosity or line parameters, we hope to remove one more bias from the quasar luminosity function (QLF).
\end{abstract}

Although the rest-frame quasar UV-optical spectrum is well-fit by a power law $\left(f(\nu) \propto \nu^{\alpha}\right.$ ) with a mean spectral index $\alpha_{U V O} \sim 0.3$ (Francis et al. 1993 ApJ 407 $519)$, this is not a sufficient description for the purposes of calculating the QLF. The QLF is 'blurred' by the range in spectral index (Warren et al. 1994 ApJ in press) and a flux-limited sample reaches deeper into the blue QLF, mimicing faster 'evolution'.

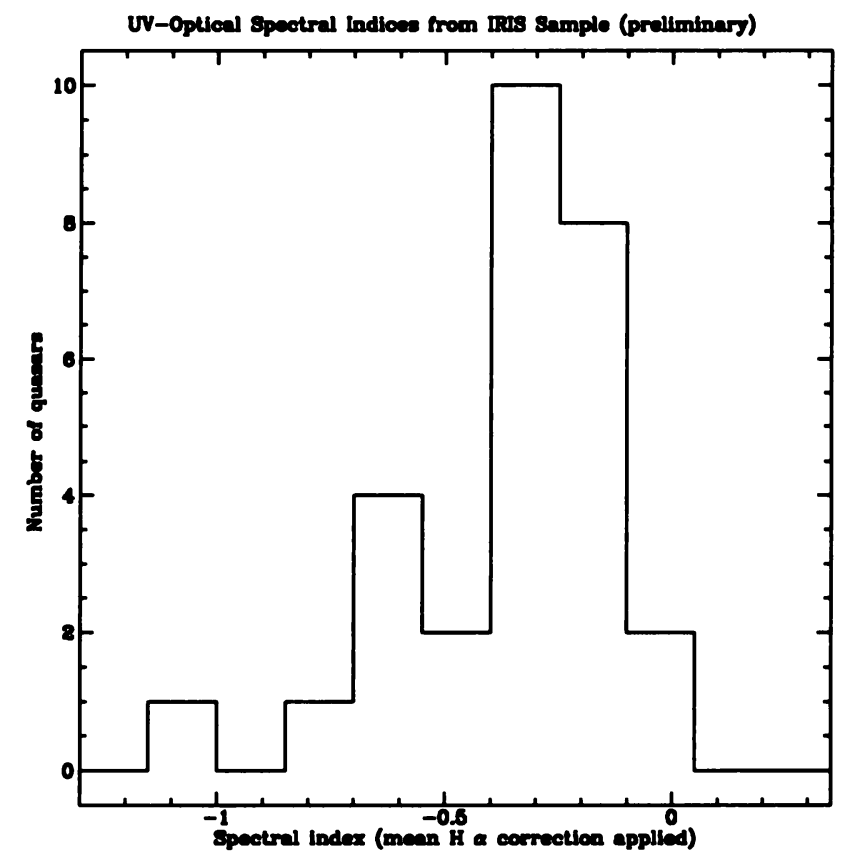

To combine with our existing $B_{J}$ magnitudes, we have obtained JHK' photometry using IRIS on the AAT for over 100 quasars, selected from the LBQS to have a wide range in other properties. Preliminary $\alpha_{U V O}$ for the first 29 objects in the sample are shown in Figure 1 ; note the range $-1<\alpha_{U V O}<0$.

498

T. J.-L. Courvoisier and A. Blecha: Multi-Wavelength Continuum Emission of AGN, 498.

C 1994 IAU. Printed in the Netherlands. 